IRON CATALYSIS

\section{Easy ester reduction}

Angew. Chem. Int. Ed. http://dx.doi.org/10.1002/ anie.201303003 (2013)

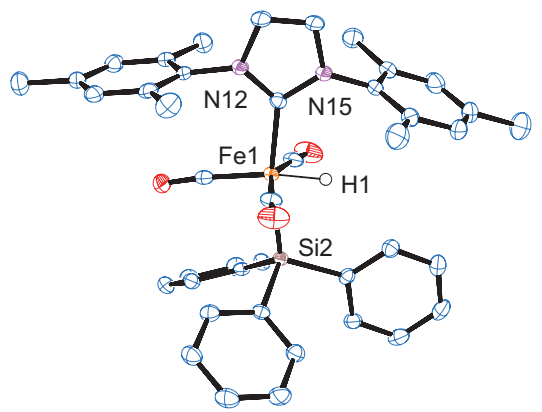

Aldehydes are useful intermediates in many synthetic schemes. Their high reactivity, however, means that they are rarely stored or carried through multiple synthetic steps in a sequence. Instead, they are often prepared immediately before use, either by oxidation or reduction of compounds at the alcohol or carboxylic-acid oxidation levels, respectively. Even then, the high reactivity of the aldehydes can be problematic because they are often found to be more reactive towards both oxidizing and reducing agents than the corresponding starting materials.

Now, Christophe Darcel, Jean-Baptiste Sortais and co-workers from Université de Rennes in France have reported that under UV irradiation several $N$-heterocyclic carbene iron carbonyl complexes can catalyse the hydrosilylation of esters. Hydrosilylation was thought to offer a promising opportunity for selective reduction of esters to aldehydes because the expected silylacetal intermediate should be stable and provide the aldehyde only on work-up of the reaction. Initial attempts at reduction using several previously reported iron catalysts gave only moderate reactivity and selectivity, so Darcel, Sortais and co-workers turned to a new class of tetracarbonyl iron $(0)$ complexes (pictured) bearing $N$-heterocyclic carbene ligands. The proposed catalytic cycle involves UV-light-mediated loss of a carbonyl ligand to give a sixteen-electron species that undergoes oxidative addition of diethylsilane to form an iron complex with both a hydride and a silyl ligand - an example of which was isolated and characterized. It is not yet clear whether the ester then reacts to insert into the $\mathrm{Fe}-\mathrm{H}$ or $\mathrm{Fe}-\mathrm{Si}$ bond, but reductive elimination from either intermediate gives the silyl acetal product and regenerates the sixteen-electron iron complex ready for the next cycle.

The reaction works at room temperature in toluene in just a few hours, and a simple acidic workup converts the silyl acetal to the aldehyde. The reaction was shown to work successfully with a variety of simple esters and with several more challenging substrates such as diesters (to give dialdehydes) and unsaturated and aromatic esters. The reduction of lactones to lactols was also shown to work with this approach.

\section{INFRARED SPECTROSCOPY}

\section{Lifetime achievement}

J.Phys. Chem. Lett. 4, 2105-2110 (2013)

Infrared (IR) spectroscopy has emerged as an important technique for monitoring the dynamic structure of biomolecules such as nucleic acids and proteins. Unlike fluorescence-based techniques in which relatively large dye molecules are attached to the analyte and monitored, infrared spectroscopy uses probe groups - such as $\mathrm{NO}, \mathrm{CO}$ and $\mathrm{CN}$ - that are much smaller and thus have less of a perturbing effect on the native structure and function of the entity being studied. Infrared probes are very sensitive to many dynamic structural features of interest, such as local electrostatics and hydrogen bonding, and advances in biochemical techniques have enabled their site-specific incorporation into biomolecules.

One such method that allows conformational changes to be monitored in peptides and proteins is to swap a natural amino acid for a synthetic one that contains an IR-active group. The probe must have certain characteristics to be of use - its IR peak must be separated from other regions of intense IR activity, its vibrational frequency should be very sensitive to local electric fields, and if it is to be used to monitor dynamics occurring on long timescales it must have a sufficiently long vibrational lifetime. Now a team led by Minhaeng Cho, Hogyu Han and Sungnam Park, from Korea University, Seoul, have shown that the lifetime of a nitrile probe can be extended by insulating it from the amino acid to which it is attached.

A nitrile group was attached to a proline derivative through either a sulfur atom, to give a $-\mathrm{SCN}$ probe group, or a selenium atom, to give a-SeCN probe. Infrared pump-probe spectroscopy was used to monitor the speed at which the vibrationally excited nitrile groups relaxed, and it was observed that the vibrational lifetime was approximately four times longer when attached through a selenium atom rather than a sulfur atom. Cho, Han, Park and colleagues suggest that the heavier selenium atom is better than sulfur at blocking the intramolecular transfer of vibrational energy from the stretching - $\mathrm{CN}$ group to the attached pyrrolidine ring, thus extending its vibrational lifetime.

Written by Gavin Armstrong, Stuart Cantrill, Stephen Davey and Anne Pichon

\section{blog $_{\text {roll }}$ 空}

\section{Fraud and favourites}

Chemists fight fabrication, compare favourites and give advice.

Thinking of faking your spectra? Think again - or at least consider carefully where you plan to send your malfeasant manuscript. Organic Letters editor Amos Smith recently penned an editorial highlighting the seriousness of data fraud: the journal has hired an analyst to inspect submissions. Perhaps unsurprisingly, Org. Lett. has found spectra that were touched up to remove evidence of impurities, and Smith has declared that corresponding authors must bear ultimate responsibility. Paul Bracher at ChemBark (http://go.nature. $\mathrm{com} / \mathrm{tGn7Eu}$ ) gives his take on Smith's editorial, noting the factors that lead to data manipulation. On the prevalence of inflated yields, Bracher notes that "part of the reason it happens is because some Pls reward it." He then contrasts the editorial with Columbia University's handling of the infamous Sezen/Sames investigation.

On a lighter note, the world of chemistry blogs has been abuzz with 'bests'. A post at ChemBark (http://go.nature.com/cAQhAq) considers the world's most influential (living) chemists. Bracher suggests Roald Hoffmann as a top choice, but notes that when it comes to engaging the public, chemists have a long way to go. Over at the Chemjobber blog (http://go.nature.com/ $m A u Z L N$ ) there is a discussion on the best advice for summer lab students/interns, in which thoughtful introspection is recommended. One commenter pointedly suggests, however, that prospective scientists "go to medical school" instead.

Finally, at Just Like Cooking (http:// go.nature.com/7X5jpR), See Arr Oh provides an analysis of what chemists say are the most important aspects of a good workplace. What things rank the highest? Meaning, fulfilment, location and great co-workers, apparently. Salary and stability are seen as less important, which is either surprising or realistic depending on one's assessment of the current chemical job landscape.

Written by Vinylogous, who blogs at http://notthelab.blogspot.com/ 